\title{
Unsupervised Image Thresholding using Fuzzy Measures
}

\author{
M Seetharama Prasad \\ Singhania University, \\ Pacheri Bari, Rajastan \\ India- 333515
}

\author{
T Divakar \\ LBRCE, \\ Mylavaram, AP \\ India - 521230
}

\author{
B Srinivasa Rao \\ LBRCE, \\ Mylavaram, AP \\ India - 521230
}

\author{
Dr C Naga Raju \\ LBRCE, \\ Mylavaram, AP \\ India - 521230
}

\begin{abstract}
Image Thresholding is a necessary task in many image processing applications. In this paper we derive fuzzy rules for $\pi$-function. We use $\pi$-function to fuzzify the original image; this is constructed to locate the intensities of the misclassification regions. Based on information theory, it maximizes the information between image foreground and background. The merit of using fuzzy set is its ability to handle uncertainty and its robustness. This technique is to optimize the image threshold by effective selection of Region Of Interest (ROI). In general Valley seeking approaches are utilized to select a threshold if the histogram is bimodal. However, histograms would not be bimodal. The fuzzy region range of the $\pi$-function is chosen as one standard deviation of the arithmetic mean $(\mu \pm \sigma)$. Because, the fuzzy region is spread on both sides of the image mean and the non-fuzzy data is located outside of this region. The limitation with the parent version is semi supervised, for low contrast images human perception is required. There exists no unsupervised appropriate procedure in literature to address this problem. The proposed method successfully segments the images of bimodal and multi-model histograms. The experimental results confirm the superiority of the proposed method over existing methods in performance. Our method produces more accurate and reliable results compared to the parent algorithm. This claim has been verified with some experimental trials using all categories of real world images.
\end{abstract}

Keywords: Segmentation, Threshold, Fuzzy measure, Region of Interest, SQC.

\section{INTRODUCTION}

Image segmentation plays a vital role in Vision and Image processing applications. It is used widely in areas such as document image analysis, scene or map processing. Satellite imaging and material inspection in quality control tasks are examples of applications that employ image thresholding or segmentation to extract useful information from images. Medical image processing is another area that has extensively used image thresholding to help the experts to better interpret digital images for a more accurate diagnosis or to plan treatment.

Segmentation based on gray level histogram thresholding is a method to divide an image containing two regions if interest; object and background. In fact, applying this threshold to the whole image, pixels whose gray level is under this value are assigned to a region and the remainder to the other. When the histogram doesn't exhibits a clear separation, ordinary thresholding techniques might perform poorly. Fuzzy set theory provides a new tool to deal with multimodal histograms.
The rest of the paper is organized as follows. Section 2 discusses about well-known segmentation methods in the literature. Section 3 explains the existing method followed by the proposed method in Section 4. Section 5 demonstrates the comparative results and at the end section 6 presents final conclusions.

\section{THRESHOLDING ALGORITHMS}

The process of segmentation separates the object from background of the image. Basically two approaches are in use for segmentation, 1. Boundary Based Approach (BBA), 2.Region Based Approach(RBA). In BBA, the boundary pixels of the object are located to separate it from the scene. Sufficient work is done in RBA, all similar pixels belongs to the object are separated from the rest of the image, 2D Gray level histogram is used to segment the image. Many popular techniques are used in this category. In ideal cases the histogram shows a deep valley between two peaks, each represents either an object or background and the threshold falls in the valley region as in fig. 1. But some images will not express clear separation of the pixels as two peaks, where threshold computation is a difficult task. To address this problem several methods have been proposed in literature [1]-[5]. Otsu [6] proposed discriminant analysis to maximize the seperability of the resultant classes. An iterative selection method is proposed in reference [7]. J.Kittler and J.Illingworth's[8] proposed minimum error Thresholding method. Entropy based algorithms proposed by Kapur et al.[9] propose a method based on the previous work of pun[10] that first applied the concept of entropy to Thresholding. His methods concludes when the sum of the background and object entropies reaches its maximum, the image threshold is obtained. In Kapur et al. Images which are corrupted with noise or irregular illumination produce multimodal histograms in which a 2D histogram does not guarantee the optimum threshold selection process, because no spatial correlation is considered. Entropy criterion function is applied on $3 \mathrm{D}$ GLSC histogram to optimize threshold by surpassing difficulties with 2D histogram [11,12]. This work is further enhanced by seetharama prasad et al.[13] with variable similarity measure. In reference [14] Type-2 fuzzy is used with GLSC histogram with human visual nonlinearity characteristics. The ordinary Thresholding techniques perform poorly where, non-uniform illumination corrupts object characteristics and inherent Image vagueness is present. Fuzzy based Image Thresholding methods are introduced in literature to overcome this problem. Fuzzy set theory [15] is 
used in these methods to handle grayness ambiguity and vagueness during the process of threshold selection. Several segmentation algorithms based on fuzzy sets are found in the literature [16]-[20]. Fuzzy clustering ideas for thresholding are in focus[21]-[23], used fuzzy memberships based on pixels distance from each class's mean to define which class a pixel belongs to and subsequently define the threshold as the cross over point of membership functions. Several segmentation algorithms based on fuzzy sets are found in the literature based on Fuzzy measure, which is a measure of vagueness in the image used in many segmentation algorithms[24]-[27], the gray level intensity value is selected to be the optimum threshold at which the fuzzy index is minimized. Haung and wang [28] assigns a membership degree to each pixel in the image, and the image is considered as a fuzzy set and the membership distribution explains each pixel belongs to either objet set or background set in the misclassification region of the histogram. Rosenfeld's convex hull method is based on analyzing the concavity structure of the histogram defined by its convex hull [29]. When the convex hull of the histogram is calculated, the deepest concavity points become candidates for the threshold value. A variation of this method can be found in [30]. Research performed by Tizhoosh et al.[31], the authors introduced opposition-based fuzzy thresholding, called OFT henceforward, and combine the concepts of fuzzy memberships and opposition- based computing to extract some local information of the image that leads to selecting a threshold value.

Anyway the key point is how to minimize the misclassification rate of the pixels and locate the correct threshold. Nuno Vieira Lopes et al.[27] proposed two statistical parameters $\mathrm{P}_{1}$ and $\mathrm{P}_{2}$ based on 30 test images to compute the effective region of interest, but the approach could not guarantee the effective selection of ROI in many cases. It requires histogram equalization in case of low contrast image; since there is no standard procedure to know

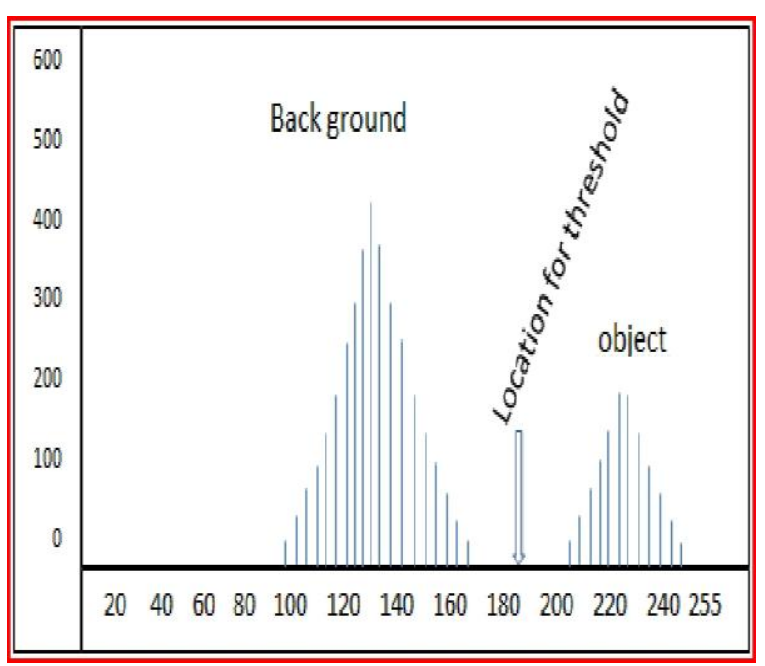

Fig 1: Bi-model image histogram and expected threshold location the contrast level of the given image it requires histogram supervision. This makes the existing technique inferior to proposed technique.

Fuzzy set theory assigns a membership degree to all elements in the universe of discourse the image $\mathrm{X}$, to the object set as well as to the background set. $\mu_{\mathrm{A}}\left(\mathrm{X}_{\mathrm{i}}\right)$ is the membership degree between 0 and 1 . Fuzzy set

$$
\mathrm{A}=\left\{\left(\mathrm{X}_{\mathrm{i}}, \mu_{\mathrm{A}}\left(\mathrm{X}_{\mathrm{i}}\right) \mathrm{X}_{\mathrm{i}} \quad \mathrm{X}\right\}\right.
$$

Where $\mathrm{X}$-is the image and $\mathrm{X}_{\mathrm{i}}$ an element of $\mathrm{X}$.

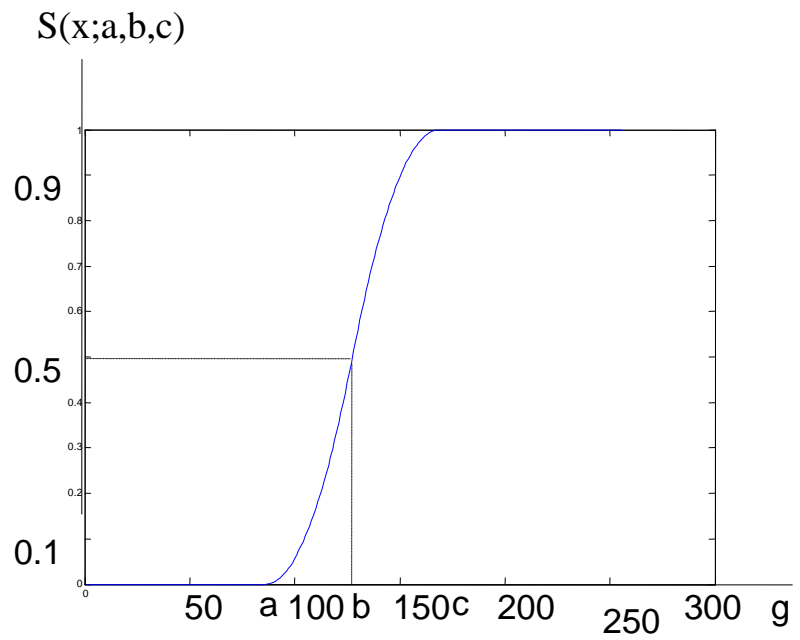

Fig 2: Shape of the S-function

The S- function is used for modeling the membership degrees as shown in Fig 2. For object pixels

$$
\begin{aligned}
& \mu_{A}(\mathrm{x})=\mathrm{S}(\mathrm{x} ; \mathrm{a}, \mathrm{b}, \mathrm{c}) \\
& =\left\{\begin{array}{lr}
0 & x<a \\
2\left\{\frac{x-a}{c-a}\right\}^{2} & a \leq x \leq b \\
1-2\left\{\frac{x-c}{c-a}\right\}^{2} & b \leq x \leq c \\
1 & x \geq c
\end{array}\right.
\end{aligned}
$$

Where $b=(a+c) / 2$, initial seed sets $a$ and $c$ controls $S$ function.

\subsection{Calculation of Parameters P1 and P2}

To begin the process the initial seed subsets are identified automatically by a procedure with statistical parameters P1 and P2. To estimate these parameters a 30 test images are considered, on which $\mathrm{P} 1$ is chosen to ensure that both the indices of fuzzy of the subsets $\mathrm{W}$ and $\mathrm{B}$ providing an increasing monotonic behavior. If $\mathrm{P} 1$ is too high the fuzzy region between the initial intervals is too small and the values are gray levels for thresholds are limited, on other hand if the $\mathrm{P} 1$ is too low the initial subsets are not representative and the 
method does not converge. Finally the P1 is estimated as the mean () value of the ensured P1 for each image among 30 test images which exhibits a significant contrast and obtained P1 value as $39.64 \%$ with standard deviation $13.37 \%$.

On other hand a $\mathrm{P} 2$ value is estimated to which is $\mathrm{P} 1$ not suitable. To determine the value of $\mathrm{P} 2$ the same procedure is used to calculate P1 and P2 value is estimated as $20 \%$ with standard deviation $14.30 \%$.

\subsection{Calculation of initial seed values}

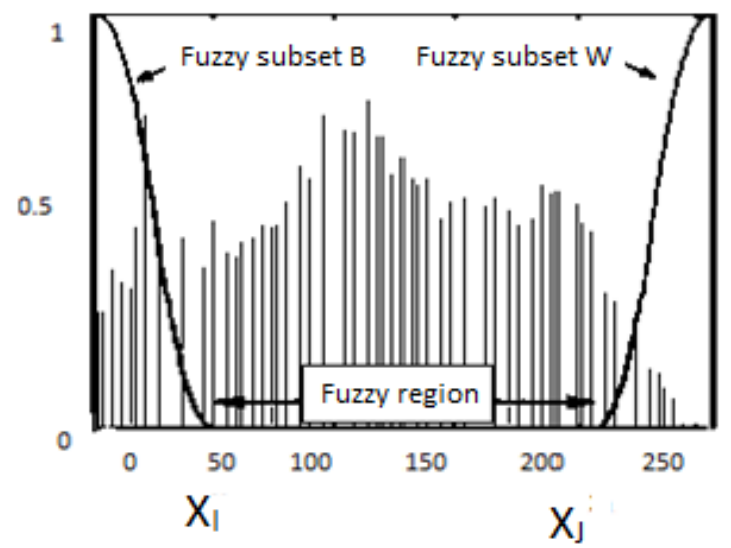

Fig 3: Multimodal image histogram and the characteristic functions for the seed subsets.

The minimum number of pixels of each set i.e object or background depends on the shape of the histogram and it is a function of the number of pixels in the gray level intervals $[0,127]$ and $[128,255]$.

$$
\text { MinPix Bseed }(\text { Oseed })=\text { P1 } \sum_{i=0(128)}^{127(255)} h\left(x_{i}\right) \text {------(3) }
$$

Where P1€ [0,1] and $\mathrm{h}\left(\mathrm{x}_{\mathrm{i}}\right)$ denotes number of occurrences at gray level $\mathrm{x}_{\mathrm{i}}$. For low contrast images popular histogram equalization is performed to bring minimum number of pixels into the region with poor number of pixels. However in the image with low contrast the method performs poorly due to the fact that one of the initial region is skewed.

Where $\mathrm{k}=1$ or 2 and $\mathrm{n}$ - number of elements in $\mathrm{A}, \mathrm{k} \epsilon$ $[1, \infty]$. The lower index of fuzziness indicates low ambiguity among elements. The image histogram is split into two crisp subsets Object $\mathrm{O}$ and back ground $\mathrm{B}$ using the measure of fuzziness. The index of fuzziness for object $\Psi(\mathrm{O})$ and background $\Psi(\mathrm{B})$ are computed using equation -(6). The normalization factor

$$
\alpha=\frac{\Psi(0)}{\Psi(B)}
$$

The normalization operator provides both initial subsets that have same index of fuzziness as in Fig. 4. The segmentation algorithm described for light object is
If the number of pixels belonging to either side of the histogram from the intensity 128 is smaller than $\mathrm{P}_{\min }=\mathrm{P} 2$ $\mathrm{MN}$, Where $\mathrm{P} 2 \in[0,1]$ and $\mathrm{M} \times \mathrm{N}$ are the total number of pixels in the image, then histogram equalization is recommended.

The initial seed subsets boundaries a and $\mathbf{c}$ values are calculated from the Equation -(3). this region is represented in Fig. 3 with boundaries $\mathbf{x}_{\mathbf{i}}$ and $\mathbf{x}_{\mathbf{j}}$

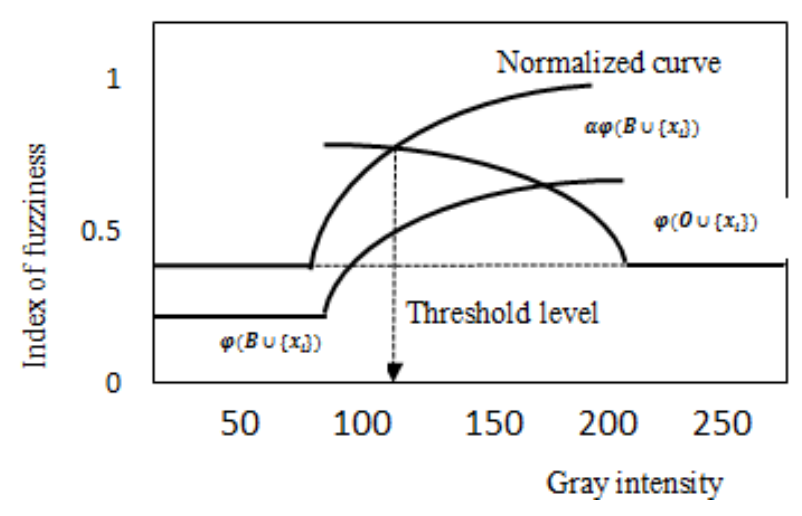

Fig 4: Normalization step and determination of the threshold value

\subsection{Measure of fuzziness}

Measure of fuzziness is the average fuzziness estimated in the image. The fuzziness of the crisp set should be zero as there is no ambiguity that the element belongs to the Object set or not. The crisp set is computed from the fuzzy set by

$\mu_{\mathrm{A}} *(\mathrm{x})=\left\{\begin{array}{l}0 \text { if } \mu_{A}(x)<0.5 \\ 1 \text { if } \mu_{A}(x) \geq 0.5\end{array}\right.$

The index is calculated by measuring the normalized distance between A calculated using equation -(2) and $\mathrm{A}^{*}$ calculated using equation -(4) as

$$
\Psi_{\mathrm{k}}(\mathrm{A})=\frac{2}{n^{1 / k}}\left[\sum_{i=1}^{n} \mid \mu_{A}\left(x_{i}\right)-\mu_{A}^{*}\left(x_{i}\right)^{k}\right]^{1 / k}
$$

1. Compute the normalization factor $\alpha$ using equation(6)

2. For all gray values $x_{i}$ in the fuzzy region compute $\psi$ $\left(\mathrm{B} \mathrm{U}\left\{\mathrm{x}_{\mathrm{i}}\right\}\right)$ and $\psi\left(\mathrm{OU}\left\{\mathrm{x}_{\mathrm{i}}\right\}\right)$

3. If $\psi\left(\mathrm{O} U\left\{\mathrm{x}_{\mathrm{i}}\right\}\right)$ is lower than $\alpha . \psi\left(\mathrm{B} U\left\{\mathrm{x}_{\mathrm{i}}\right\}\right)$ then $x_{i}$ is included in set $O$, otherwise $x_{i}$ is included in set $\mathrm{B}$.

\subsection{Drawbacks in the existing method}

Values of $\mathrm{P} 1$ and $\mathrm{P} 2$ are derived from 30 test images, but the approach could not guarantee the effective selection of ROI in many cases. On the other hand it requires histogram equalization in case of low contrast image; since there is no 
standard procedure to know the contrast level of the given image it requires the attention of the human histogram supervision.

\section{PROPOSED METHOD}

Due to the above drawbacks semi supervise method is necessary to find an unsupervised method . In this paper we introduce a technique for the automatic selection of seed subsets to decide the effective ROI. The fuzzy $\pi$-function produces normally distributed intensities for any given data. $\mu \pm \sigma$ from the normally distributed data, would represents all the characteristics of the data. So $68.2 \%$ of data values are equally spread on either sides of the image mean and the remaining $21.8 \%$ is scattered over extreme ends of the histogram. Fuzzy intensities mostly packed near the mean of the Image where the optimum threshold could be found. As the initial seed sets are located at one standard deviation $(\sigma)$ of both sides of the mean $(\mu)$ on the histogram.

\subsection{ROI Computation}

Broadly we can divide images into three categories such as i. object dominant, ii. Background dominant and iii. equally distributed as shown from left to right respectively in Fig 5. However in all three cases the arithmetic mean $(\mu)$ of the image gives a clue to locate ROI where as standard deviation $(\sigma)$ decides the boundaries.
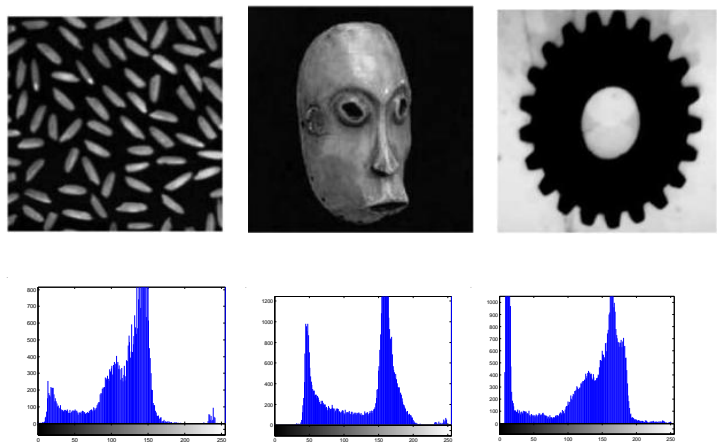

Fig 5: Three image categories and corresponding histograms.

Let $x(\mathrm{i}, \mathrm{j})$ be the gray level intensity of image at $(\mathrm{i}, \mathrm{j}) . \mathrm{I}=\{$ $x(\mathrm{i}, \mathrm{j}) \mid \mathrm{i \epsilon}[1, \mathrm{Q}], \mathrm{j} \epsilon[1, \mathrm{R}]\}$ is an image of size $\mathrm{Q} \times \mathrm{R}$, i.e. $\mathrm{N}$. The gray level set $\{0,1,2, \ldots . .255\}$ is considered as $G$ throughout this method for convenience. The mean $(\mu)$ and standard $\operatorname{deviation}(\sigma)$ are calculated as follows

$$
\begin{aligned}
& \mu=\frac{1}{N} \sum_{i=1}^{n} x_{i} \times h(i) \\
& \sigma=\sqrt{\frac{1}{N} \sum_{i=1}^{n}\left(x_{i}-\mu\right)^{2}}
\end{aligned}
$$

From the Fig. 5 it is clear that in any category of image the mean lies in the range of dominant pixels of the image, or between the two or more dominant pixels region, hence mean evidences the
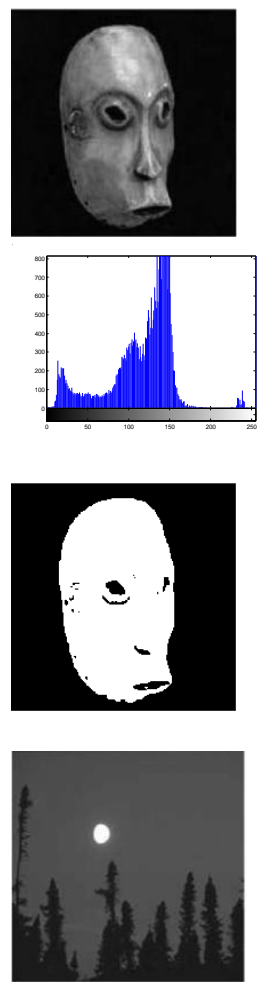
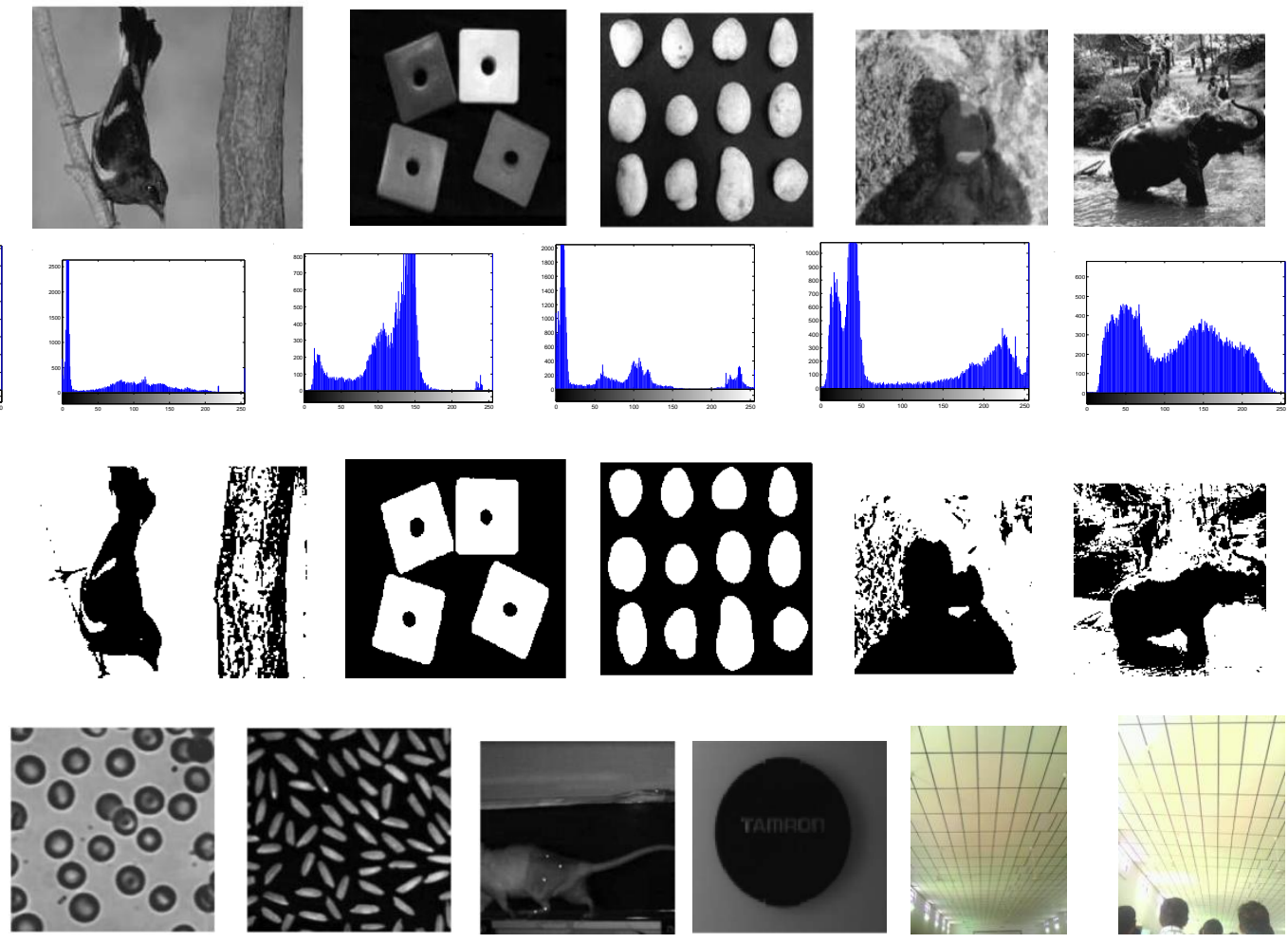

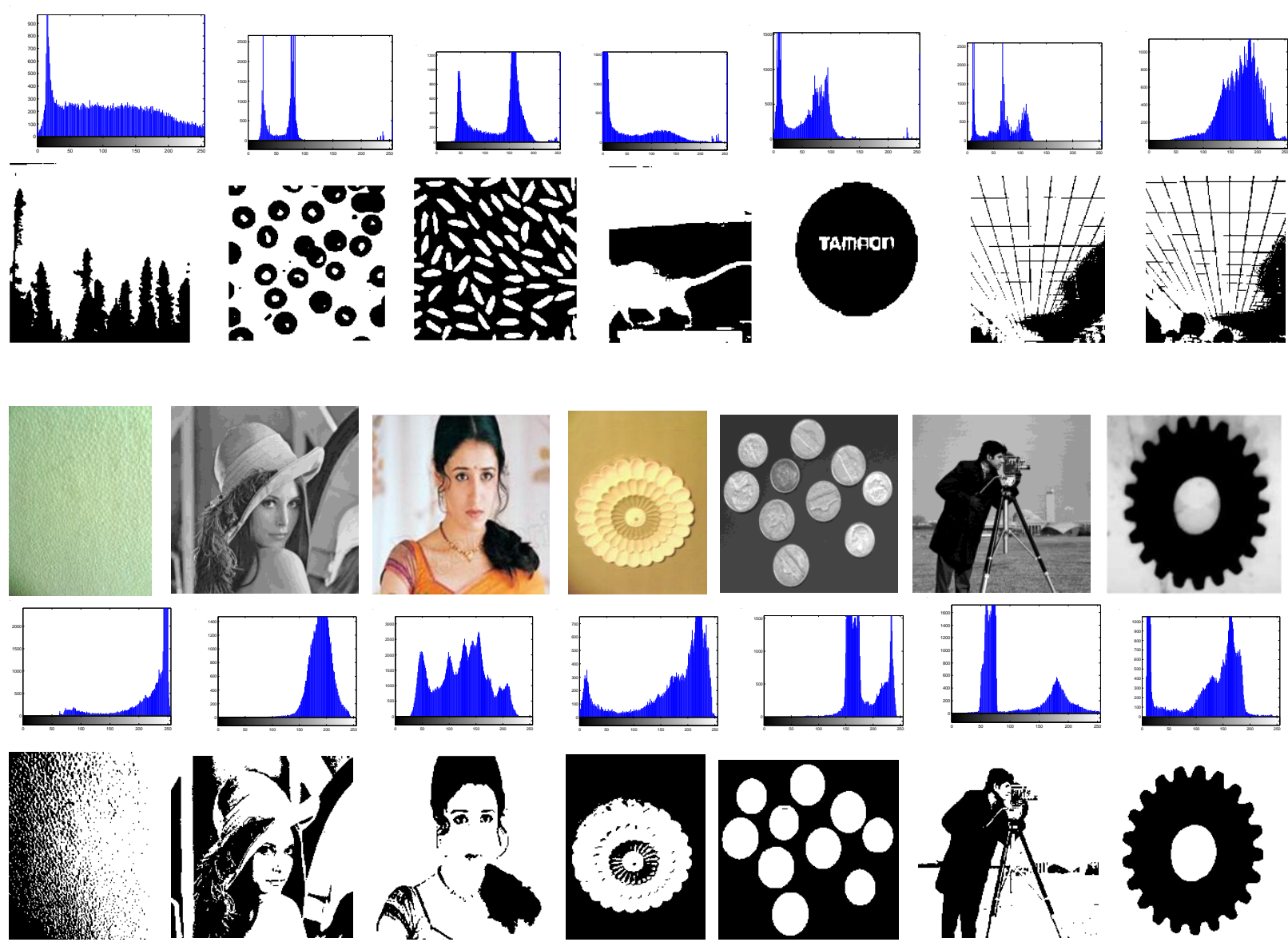

Fig 6: Test images and their corresponding histograms and Ground Truth Images ( from top to down )

location of object or back ground. The key of the proposed method is to optimize the range of ROI, because the vagueness in the image is always lies around the edges. Hence the best selection of ROI should always include the above said fuzzy region. The standard deviation $(\sigma)$ will limit the fuzzy region on gray scale histogram of the image from the mean $(\mu)$ on both sides.

\subsection{The Fuzzy $\pi$-function}

The proposed method eliminates the assumptions of initial seed subsets of $\boldsymbol{\pi}$-function as shown in Fig.7. Here we consider a fuzzy $\pi$-function[34] to transform the fuzzy intensities into normally distributed intensities. The $\mathrm{p}$ and $\mathrm{q}$ values are derived as follows to optimize ROI selection. The intensities of an image are transformed to an interval $[0,1]$ by $\boldsymbol{\pi}$-function in terms of a standard S-function using equation (9). The values of the function represent the degrees of the closeness in terms of intensities. The function is, therefore, used to locate the intensities of object and background. The selection of a cross-over point which is the arithmetic mean of the image could be viewed as an object-background classification problem. The fuzzy region of the function is chosen as the range from the mean intensity to the standard deviation of the whole image, since the proposed approach assumes that the fuzzy intensities exists within one standard deviation from the mean. It implies that the fuzzy intensities of are located somewhere around the mean intensity of an image.

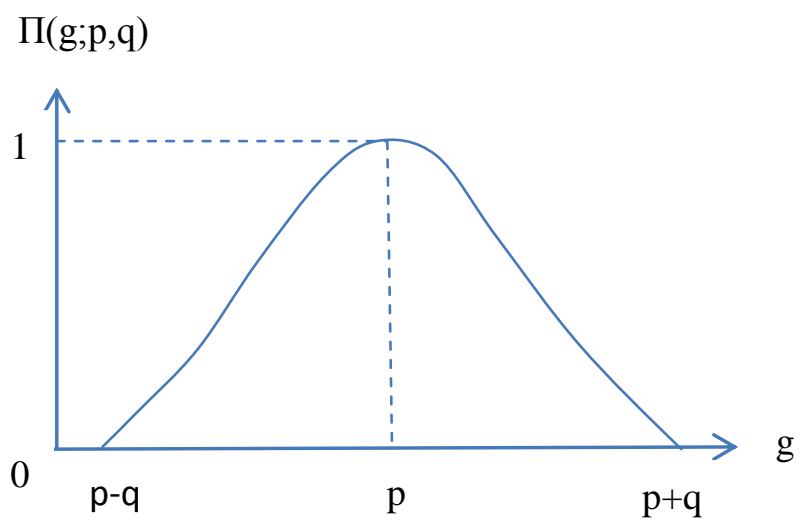

Fig 7: fuzzy $\pi$ function

$$
\begin{aligned}
\mu_{\pi}(\mathrm{x}) & =\boldsymbol{\pi}(\mathrm{x} ; \mathrm{p}, \mathrm{q}) \\
& = \begin{cases}\boldsymbol{s}\left(\boldsymbol{x}: \boldsymbol{p}-\boldsymbol{q}, \frac{\boldsymbol{p}-\boldsymbol{q}}{2}, \boldsymbol{q}\right), & \text { if } \boldsymbol{x} \leq \boldsymbol{p} \\
1-\boldsymbol{s}\left(\boldsymbol{x}: \boldsymbol{p}-\boldsymbol{q}, \frac{\boldsymbol{p}-\boldsymbol{q}}{2}, \boldsymbol{q}\right), & \text { if } \boldsymbol{x}>p\end{cases}
\end{aligned}
$$

Where, $\mathrm{p}=\mu$ and $\mathrm{q}=\sigma$ 


\subsection{Algorithm for Segmentation}

STEP1. From the $\boldsymbol{\pi}$-curve compute membership degrees of each intensity value using equation -(9). $\mathrm{p}$ and $\mathrm{q}$ values are calculated from equations $-(7)$ and $-(8)$ respectively.

STEP 2. The index of fuzziness for the object $\Psi(\mathrm{O})$ and the background $\Psi(\mathrm{B})$ are computed using equation -(5). The normalization factor from Equation -(6).

STEP 3. The segmentation algorithm described for light object is
A. Compute the normalization factor $\alpha$
B. For all gray values $x_{i}$ in the fuzzy region compute $\psi\left(\mathrm{B} \mathrm{U}\left\{\mathrm{x}_{\mathrm{i}}\right\}\right)$ and $\psi\left(\mathrm{O} U\left\{\mathrm{x}_{\mathrm{i}}\right\}\right)$
C. If $\psi\left(\mathrm{OU}\left\{\mathrm{x}_{\mathrm{i}}\right\}\right)$ is lower than $\alpha . \psi\left(\mathrm{B} U\left\{\mathrm{x}_{\mathrm{i}}\right.\right.$ \}) then $x_{i}$ is included in set $O$, otherwise $x_{i}$ is included in set $B$.

\section{RESULTS AND DISCUSSION}

After all the above discussion on the proposed technique, we now present the performance of this methodology in this section. In order to evaluate this 20 real time images are considered with variety of image characteristics from uni model to multi model histograms. For each sample image its histogram and a ground truth image, which is generated manually has been used as gold standard are shown in Fig. 6, to compare results and their performance. We undertake this process with two measures similarity and diversity between the output image and it's corresponding ground truth image.
Table 1. Efficiency comparison using Jaccard

Index

\begin{tabular}{|c|c|c|c|}
\hline \multirow{2}{*}{ IMAGE } & \multicolumn{3}{|c|}{ Jaccard Index $(\%)$} \\
\hline & Proposed & Parent & Otsu \\
\hline zimbo & 99.93 & 97.76 & 96.50 \\
\hline bird & 96.75 & 96.75 & 89.07 \\
\hline blocks & 97.65 & 73.67 & 71.90 \\
\hline patotoes & 99.94 & 86.12 & 97.39 \\
\hline shadow & 97.17 & 85.26 & 95.45 \\
\hline forest & 98.45 & 89.76 & 89.43 \\
\hline trees & 98.81 & 35.72 & 30.73 \\
\hline blood cells & 98.24 & 88.37 & 98.91 \\
\hline rice & 97.91 & 86.44 & 97.60 \\
\hline animal & 96.29 & 83.49 & 51.84 \\
\hline TAMRON & 95.67 & 73.77 & 66.46 \\
\hline roof & 81.68 & 79.50 & 80.52 \\
\hline rf_heads & 92.91 & 77.38 & 85.23 \\
\hline Sheet & 90.12 & 62.76 & 98.07 \\
\hline Leena & 93.64 & 86.83 & 91.44 \\
\hline Anshu & 87.02 & 91.11 & 96.92 \\
\hline wl_flower & 99.67 & 35.94 & 95.56 \\
\hline Coins & 99.82 & 87.47 & 97.35 \\
\hline camera man & 97.45 & 99.18 & 99.61 \\
\hline gear wheel & 99.96 & 91.97 & 99.54 \\
\hline $\operatorname{mean}(\mu)$ & 95.95 & 80.46 & 86.48 \\
\hline $\operatorname{std}(\sigma)$ & 4.81 & 17.62 & 18.30 \\
\hline
\end{tabular}

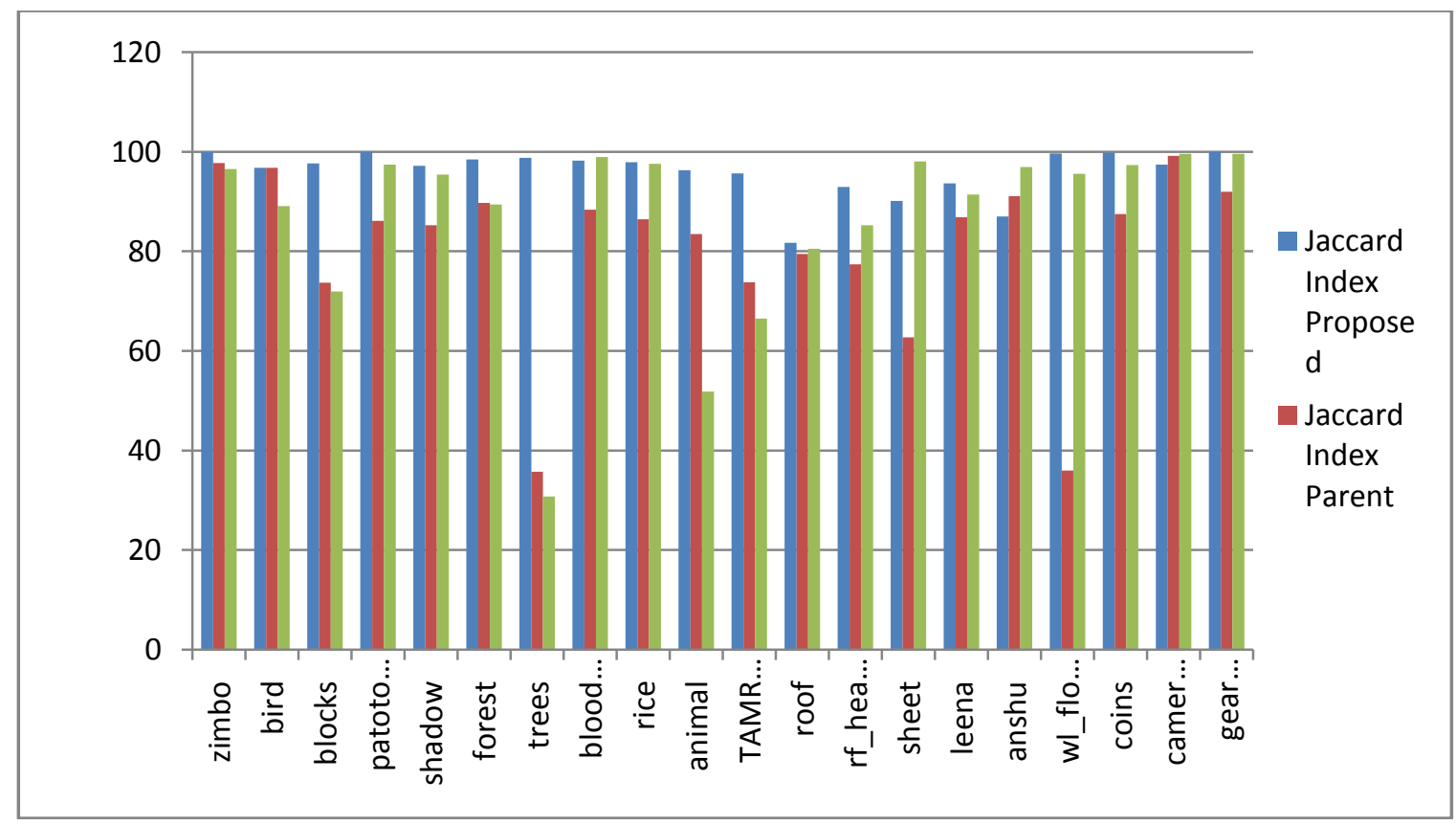

Fig 8: Efficiency comparison using Jaccard Index 
The first measure similarity is the Jaccard Index [32] known as Jaccard similarity coefficient, very popular and used mostly as similarity indices for binary data. The area of overlap $A_{j}$ is calculated between the thresholded binary image $B_{j}$ and its corresponding gold standard image $G_{j}$ as shown in equation -(10).

$$
A_{i}=\frac{\left|B_{i} \cap G_{i}\right|}{\left|B_{i} \cup G_{i}\right|}
$$

If the thresholded object and corresponding gold standard image $\mathrm{G}_{\mathrm{j}}$ (associated ground truth image) are exactly identical then the measure is 1 and the measure 0 represents they are totally disjoint, but the higher measure indicates more similarity. Jaccard index of the proposed method is compared with the parent algorithm and the standard Otsu's technique and shown in Table. 1 and the Fig. 8 demonstrate the superiority of the proposed method.

The other method Dice coefficient[33] which is another similarity measurement related to Jaccard Index method is as follows, In order to evaluate the performance, two measurements of similarity and diversity between the result image and its associated gold standard image are considered as shown in equation -(11)

$$
D_{i}=\frac{2\left|B_{i} \cap G_{i}\right|}{\left|B_{i}\right|+\left|G_{i}\right|}
$$

Again with this measure, a value of zero indicates no overlap while 1 shows a perfect match between the resulted image and

Table 2. Efficiency comparison using Dice coefficient

\begin{tabular}{|c|c|c|c|}
\hline animal & 92.85 & 71.65 & 34.99 \\
\hline TAMRON & 91.70 & 58.44 & 49.77 \\
\hline roof & 69.03 & 65.97 & 67.40 \\
\hline rf_heads & 86.76 & 63.11 & 74.27 \\
\hline sheet & 82.01 & 45.73 & 96.21 \\
\hline leena & 88.04 & 76.73 & 84.23 \\
\hline anshu & 77.02 & 83.68 & 94.02 \\
\hline wl_flower & 99.35 & 21.91 & 91.49 \\
\hline coins & 99.64 & 77.73 & 94.83 \\
\hline camera man & 95.02 & 98.38 & 99.22 \\
\hline gear wheel & 99.93 & 85.13 & 99.08 \\
\hline $\operatorname{mean}(\mu)$ & 92.59 & 70.22 & 79.68 \\
\hline $\operatorname{std}(\sigma)$ & 8.30 & 21.04 & 23.13 \\
\hline
\end{tabular}

\begin{tabular}{|c|c|c|c|}
\hline \multirow{2}{*}{ IMAGE } & \multicolumn{3}{|c|}{ Dice Coefficient (\%) } \\
\cline { 2 - 4 } & Proposed & Parent & Otsu \\
\hline zimbo & 99.85 & 95.61 & 93.23 \\
\hline bird & 93.71 & 93.71 & 80.29 \\
\hline blocks & 95.40 & 58.32 & 56.13 \\
\hline patotoes & 99.88 & 75.62 & 94.90 \\
\hline shadow & 94.50 & 74.31 & 91.29 \\
\hline forest & 96.94 & 81.42 & 80.89 \\
\hline trees & 97.64 & 21.74 & 18.16 \\
\hline blood cells & 96.55 & 79.17 & 97.85 \\
\hline rice & 95.90 & 76.12 & 95.32 \\
\hline
\end{tabular}

its ground truth image. As it is very clear, this process is similar to Jaccard but gives twice the weight to agreements. Furthermore, by applying both of these measures to resulted images, the goal is to get as close to 1 as possible. Dice coefficient is computer for the proposed method along with the parent algorithm and the Otsu's technique in Table. 2 , the significance of the proposed method is exhibited in Fig. 9.

From the experiments for each test image we obtain Jaccaard Index values [32] computed from equation -(10) is compared with gold standard images by all three different methods including Proposed, Parent and OTSU's are in TABLE 1. The proposed method surpasses both the other methods by demonstrating mean $(\mu)$ as $95.95 \%$ and standard deviation $(\sigma)$ as $4.81 \%$. The second measurement Dice coefficient [33] is also computed from equation -(11) for each test image and compared with its gold standard images and obtained values as shown in TABLE 2 for all three methods. The proposed method exhibits clear lead by keeping the mean $(\mu)$ and standard deviation $(\sigma)$ are as $92.59 \%$ and $8.30 \%$ respectively. The proposed method confirms the qualitative improvement over the existing methods. Fig. 10 shows threshold results obtained by various techniques. 


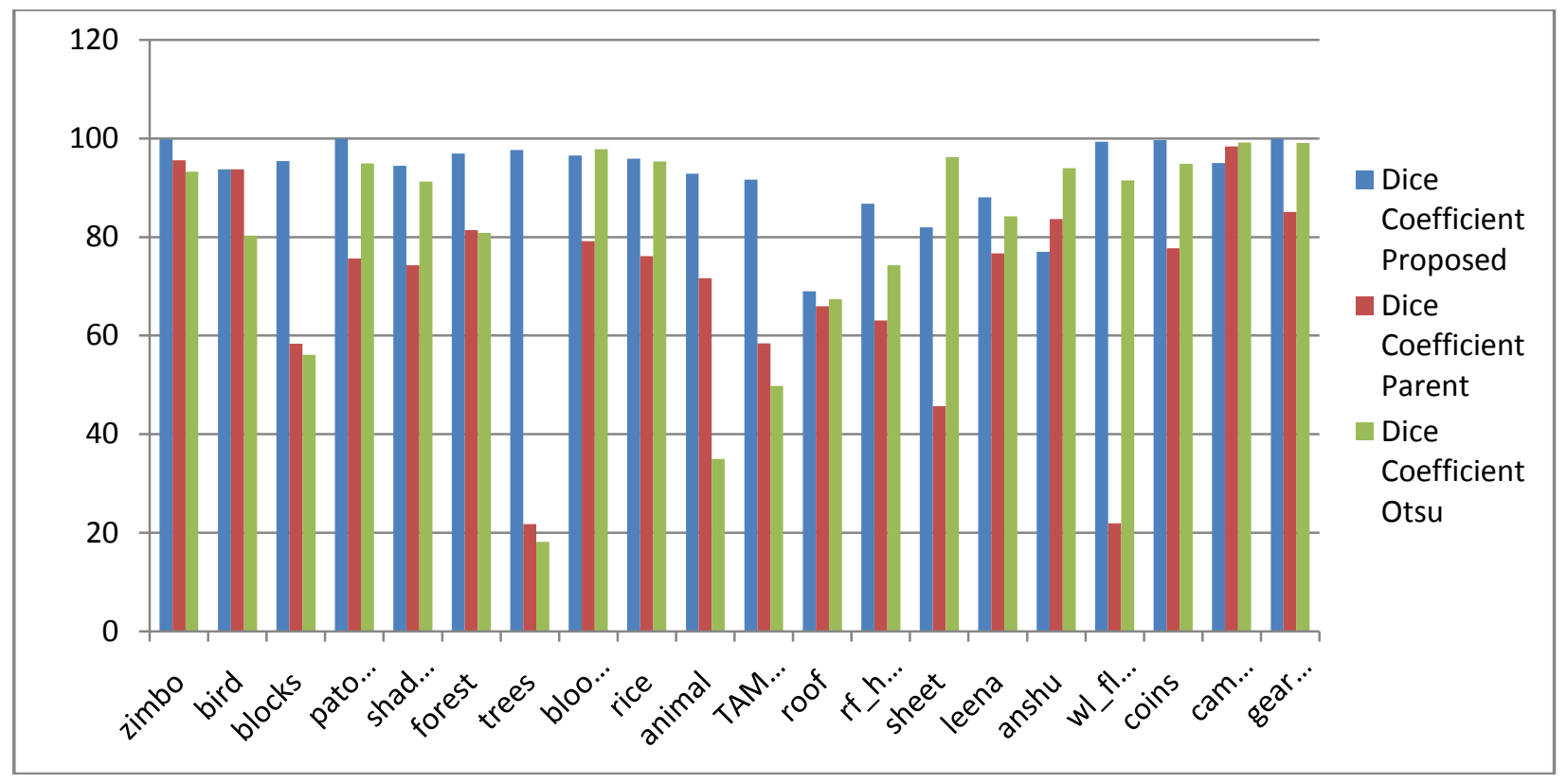

Fig 9: Efficiency comparison using Dice coefficient

\section{CONCLUSION}

4.3 In this paper, based on Fuzzy index of fuzzy measure an automatic histogram threshold approach is presented. This work overcomes the existing problems with the parent method related to finding the Region of interest, in this case the fuzzy region of the given image and finalizing the initial seed subsets, the boundaries of the fuzzy region. The fuzzy $\pi$-function would yield the given data to a normally distributed data from which the fuzzy seed subsets
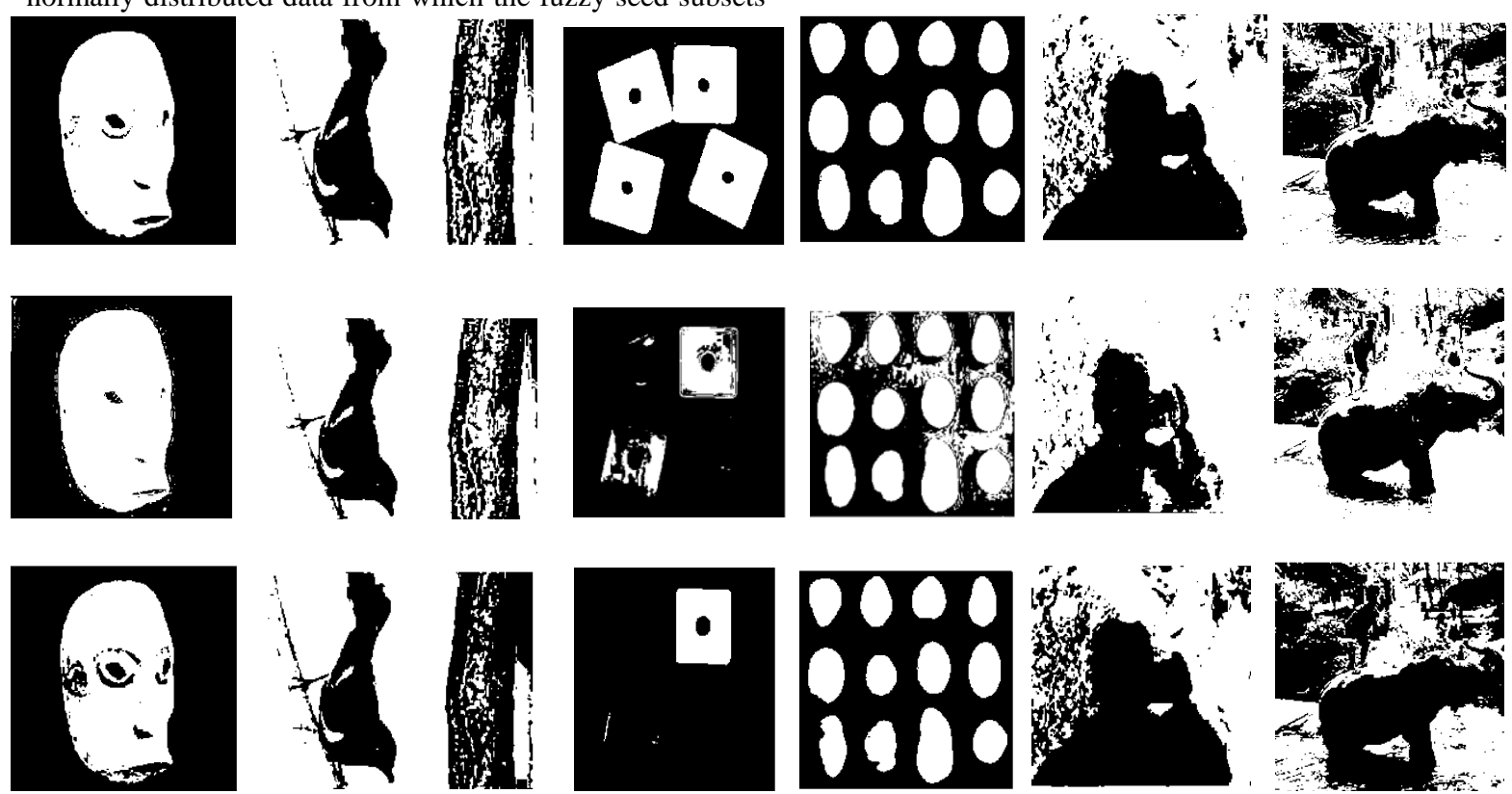
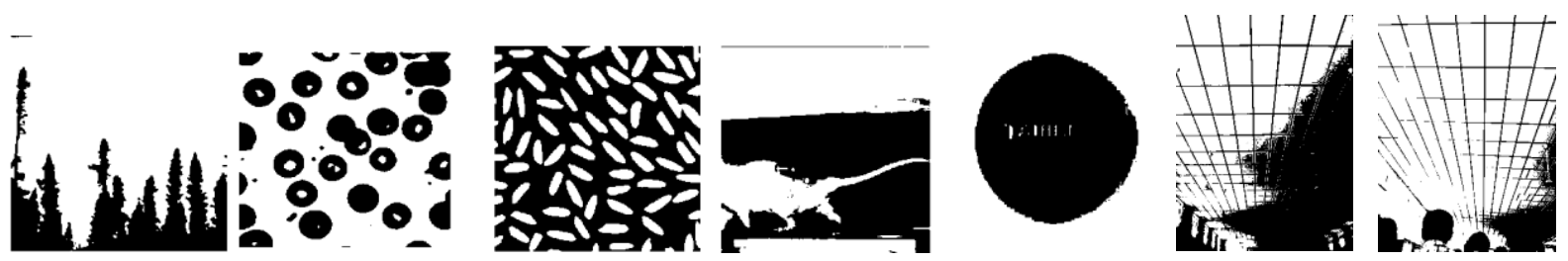

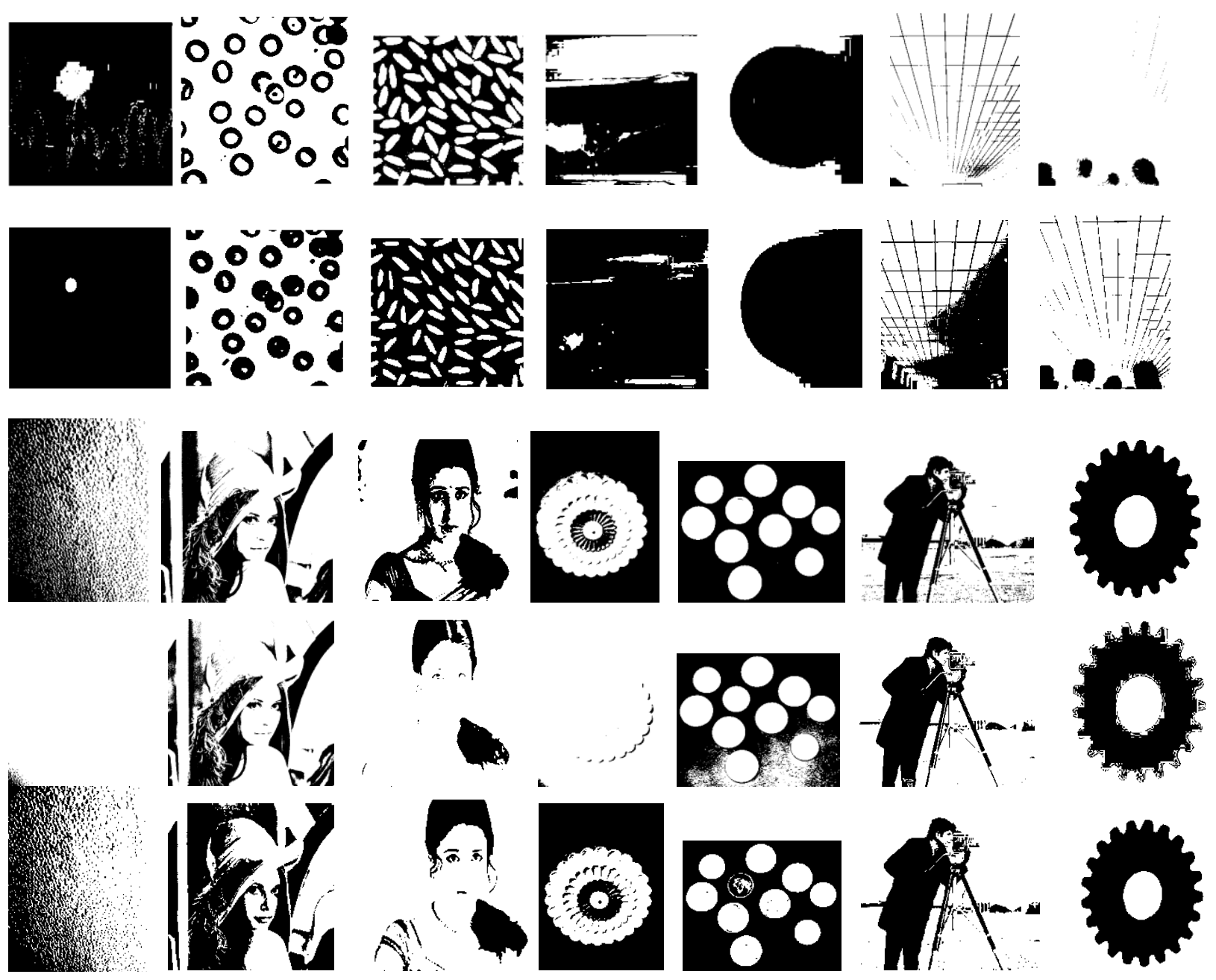

Fig 10: Resultant se gmented images. For each image, from top to bottom: proposed, parent and standard Otsu technique.

well. Here we conclude that this procedure presents a higher performance for all types of images. In future, this work, can be extended with other fuzzy measures like Shannon's entropy and Yager's fuzzy measure to test which fuzzy measure works best for what type of images. The proposed

\section{ACKNOWLEDGMENTS}

We thank the Chancellor, Vice chancellor, Research cell, Department of Computer Science of Singhania University, Rajastan, India and Director, Management of

\section{REFERENCES}

[1] W. K. Pratt, Digital Image Processing, third ed. New York: Wiley, 2001.

[2] R. C. Gonzalez and R. E.Woods, Digital Image Processing. Reading, MA: Addison-Wesley, 1993.

[3] N. R. Pal and S. K. Pal, "A review on image segmentation techniques", pattern recog.,vol.26,No. 9, pp.1277-1294,1993. technique is estimating the region of interest, the fuzzy region of the image better than any existing unsupervised technique. still there is a room for improvise this method by the application of statistical quality control (SQC) on the normally distributed data.

Lakireddy Balireddy College of Engineering, Mylavaram, India for their continuous support.

[4] Y.J. Zhang, "A survey on evaluation methods for image segmentation," Computer Vision and Pattern Recognition, vol. 29, pp. 1335-1346, 1996.

[5] M. Sezgin and B. Sankur, "Survey over image thresholding techniques and quantitative performance evaluation," J. Electron. Imag., vol. 13, no. 1, pp. 146-165, Jan. 2004. 
[6] N. Otsu, "A threshold selection method from gray level histograms," IEEE Trans. Syst., Man, Cybern., vol. SMC-9, pp. 62-66, 1979.

[7] T. Ridler and S. Calvard, "Picture thresholding using an iterative selection method," IEEE Trans. Syst., Man, Cybern., vol. SMC-8, pp. 630-632, Aug. 1978.

[8] J. Kittler and J. Illingworth, "Minimum error thresholding," Pattern Recognit., vol. 19, no. 1, 1986.

[9] J. N. Kapur, P. K. Sahoo, and A. K. C.Wong, "A new method for graylevel picture thresholding using the entropy of the histogram," Graph.Models Image Process., vol. 29, pp. 273-285, 1985.

[10] T. Pun, "A new method for gray-level picture thresholding using the entropy of the histogram," Signal Process., vol. 2, no. 3, pp. 223-237, 1980.

[11] Yang Xiao, Zhiguo Cao, Tianxu Zhang "Entropic thresholding based on gray level spatial correlation histogram", IEEE trans. $19^{\text {th }}$ international conf., pp. 14,ICPR-2008.

[12] Y.Xiao, Z.G.Cao, and S.Zhong, "New entropic thresholding approach using gray-level spatial correlation histogram", Optics Engineering, 49, 127007,2010

[13] M Seetharama Prasad, T Divakar, L S S Reddy, "Improved Entropic Thresholding based on GLSC histogram with varying similarity measure", International Journal of Computer Applications, vol.24, June 2011

[14] Yang Xiao, Zhiguo Cao,Wen zhuo, "Type-2 fuzzy thresholding using GLSC histogram of human visual nonlinearity characteristics", Optics Express, vol.19, no.11, 10657, May 2011.

[15] L.A.Zadeh,’Fuzzy sets”, Inf. Control 8, 338-353,1965

[16] C. Murthy and S. Pal, "Fuzzy thresholding: Mathematical framework, bound functions and weighted moving average technique," Pattern Recognit. Lett., vol. 11, pp. 197-206, 1990.

[17] O. J. Tobias, R. Seara, and F. A. P. Soares, "Automatic image segmentation using fuzzy sets," in Proc. $38^{\text {th }}$ Midwest Symp. Circuits and Systems, 1996, vol. 2, pp. 921-924.

[18] O. J. Tobias and R. Seara, "Image segmentation by histogram thresholding using fuzzy sets," IEEE Trans. Image Process., vol. 11, 2002.

[19] A. S. Pednekar and I. A. Kakadiaris, "Image segmentation based on fuzzy connectedness using dynamic weights," IEEE Trans. Image Process., vol. 15, no. 6, pp. 1555-1562, Jun. 2006.

[20] F. Sahba and H.R. Tizhoosh, "Quasi-Global Oppositional Fuzzy Thresholding" in Proc. IEEE International Conference on Fuzzy Systems (FUZZIEEE), Korea, August 20-24, 2009, pp. 1346-1351.
[21] C. V. Jawahar, P. K. Biswas, and A. K. Ray, "Investigations on fuzzy thresholding based on fuzzy clustering", Pattern Recogn. 30(10) pp. 1605-1613,1997.

[22] K. S. Chuang, H. L. Tzeng, S. Chen, J. Wu, and T. J. Chen, "Fuzzy c-means clustering with spatial information for image segmentation," Comput. Med. Imag. Graph., vol. 30, no. 1, pp. 9-15, 2006.

[23] S. Sahaphong and N. Hiransakolwong, "Unsupervised image segmentation using automated fuzzy c-means," in Proc. IEEE Int. Conf. Computer and Information Technology, pp. 690-694, Oct. 2007.

[24] A. Kaufmann, Introduction to the Theory of Fuzzy Subsets. New York: Academic, 1975, vol. I.

[25] N. R. Pal and J. C. Bezdek, "Measuring fuzzy uncertainty," IEEE Trans.Fuzzy Syst., vol. 2, 1994.

[26] H. R. Tizhoosh, "Image thresholding using type II fuzzy sets," Pattern Recognit., vol. 38, pp. 2363-2372, 2005.

[27] Nuno Vieira Lopes et al. "Automatic Histogram Threshold using Fuzzy Measures" IEEE Trans. Image Process., vol. 19, no. 1, Jan. 2010.

[28] L. K. Huang and M. J. J. Wang, "Image thresholding by minimizing the measures of fuzziness," Pattern Recognit., vol. 28, no. 1, pp. 41-51, 1995.

[29] A. Rosenfeld and P. de la Torre, "Histogram concavity analysis as an aid in threshold selection," $S M C$, vol. 13, no. 3, pp. 231-235, Mar. 1983.

[30] J. S. Wezka and A. Rosenfeld, "Histogram Syst., Man, Cybern., vol. SMC-9, pp. 38-52, 1979.

[31] F. Sahba and H.R. Tizhoosh, "Quasi-Global Oppositional Fuzzy Thresholding" in Proc. IEEE International Conference on Fuzzy Systems (FUZZ - IEEE), Korea, August 20-24, 2009, pp. 1346-1351.

[32] Paul Jaccard, "Etude comparative de la distribution orale dans une portion des Alpes et des Jura". In Bulletin del la Socit Vaudoise des Sciences Naturelles, volume 37, pages 547-579.

[33] Lee R. Dice, "Measures of the Amount of Ecologic Association Between Species," Ecology 26 (3): 297302,1945

[34] Heng-Da Cheng, Yui Man Lui, and Rita I.Freimanis, "A Novel Approach to Microcalcification Detection Using Fuzzy Logic Technique", IEEE TRANSACTIONS ON MEDICAL IMAGING, VOL. 17, NO. 3, pp. 442-450, June. 1998 